\title{
Direct interaction with filamins modulates the stability and plasma membrane expression of CFTR
}

\author{
William R. Thelin, ${ }^{1}$ Yun Chen, ${ }^{1}$ Martina Gentzsch, ${ }^{1}$ Silvia M. Kreda, ${ }^{2}$ Jennifer L. Sallee, ${ }^{1}$ \\ Cameron O. Scarlett, ${ }^{3}$ Christoph H. Borchers, ${ }^{3}$ Ken Jacobson, ${ }^{1,4}$ \\ M. Jackson Stutts, ${ }^{2}$ and Sharon L. Milgram ${ }^{1}$
}

${ }^{1}$ Department of Cell and Developmental Biology, ${ }^{2}$ Cystic Fibrosis Pulmonary Research and Treatment Center, ${ }^{3}$ Department of Biochemistry and Biophysics, and ${ }^{4}$ Lineberger Comprehensive Cancer Center, University of North Carolina at Chapel Hill, Chapel Hill, North Carolina, USA.

\begin{abstract}
The role of the cystic fibrosis transmembrane conductance regulator (CFTR) as a cAMP-dependent chloride channel on the apical membrane of epithelia is well established. However, the processes by which CFTR is regulated on the cell surface are not clear. Here we report the identification of a protein-protein interaction between CFTR and the cytoskeletal filamin proteins. Using proteomic approaches, we identified filamins as proteins that associate with the extreme CFTR $N$ terminus. Furthermore, we identified a disease-causing missense mutation in CFTR, serine 13 to phenylalanine (S13F), which disrupted this interaction. In cells, filamins tethered plasma membrane CFTR to the underlying actin network. This interaction stabilized CFTR at the cell surface and regulated the plasma membrane dynamics and confinement of the channel. In the absence of filamin binding, CFTR was internalized from the cell surface, where it prematurely accumulated in lysosomes and was ultimately degraded. Our data demonstrate what we believe to be a previously unrecognized role for the CFTR N terminus in the regulation of the plasma membrane stability and metabolic stability of CFTR. In addition, we elucidate the molecular defect associated with the S13F mutation.
\end{abstract}

\section{Introduction}

Cystic fibrosis (CF) is an autosomal-recessive genetic disease caused by mutations in the single gene encoding the cystic fibrosis transmembrane conductance regulator (CFTR). CFTR is normally expressed on the apical plasma membrane of epithelial cells, where it functions as a cAMP-regulated chloride channel. CF is characterized by an ion and solute transport defect that, in the lungs, culminates in accumulation of dehydrated mucus, impaired mucociliary clearance, and chronic bacterial infection. Lung disease, the major cause of morbidity in CF patients, remains a significant detriment to a healthy life for CF patients.

Since the identification of the gene encoding CFTR, more than 1,000 different disease-causing mutations have been identified in $\mathrm{CF}$ patients. CFTR mutations are functionally categorized by defects in early biosynthesis and folding (class I, II, and V), impaired chloride channel activity (class III and IV), or destabilization of the mature protein (1-3). The study of CFTR mutations has provided important insights into the regulation of virtually all aspects of CFTR biology including biosynthesis $(4,5)$, gating $(6,7)$, endocytosis $(8,9)$, and degradation $(3,10)$. Current therapeutic strategies are targeting the basic defects associated with mutant CFTR proteins with the goal of correcting these defects in vivo $(11,12)$. Consequently, the characterization of the processes by which CFTR is regulated in normal and disease states will provide critical insights into normal CFTR regulation as well as aid in the development of new treatment approaches.

Nonstandard abbreviations used: BHK, baby hamster kidney; $\mathrm{CF}$, cystic fibrosis; CFTR, cystic fibrosis transmembrane conductance regulator; FLN, filamin; MALDI, matrix-assisted laser desorption/ionization; MS, mass spectrometry; SPT, single particle tracking; TCZ, transient confinement zone; WD-HBE, well differentiated human bronchial epithelia.

Conflict of interest: The authors have declared that no conflict of interest exists. Citation for this article: J. Clin. Invest. 117:364-374 (2007). doi:10.1172/JCI30376.
Here, we studied disease-causing mutations of the cytosolic $\mathrm{N}$ terminus of CFTR in order to elucidate the functional importance of this domain. Truncation of the 80 -amino acid $\mathrm{N}$ terminus disrupts normal biosynthesis, demonstrating an absolute requirement for this domain (13). Zhang et al. identified the $\mathrm{N}$ terminus as a site of interaction for the cysteine string protein, a molecular chaperone that promotes normal CFTR folding $(14,15)$. In addition, the $\mathrm{N}$ terminus contains an arginine-framed tripeptide sequence (residues 29-31) that functions as an ER retention motif $(16,17)$. At the cell surface, CFTR is regulated by interactions with the soluble N-ethyl maleimide-sensitive factor attachment protein receptors (t-SNAREs) syntaxin 1A and SNAP23 $(18,19)$. The direct association of t-SNAREs to the CFTR $\mathrm{N}$ terminus negatively regulates channel gating as well as membrane trafficking (18-24). CFTR channel activity can also be regulated through an intramolecular interaction between the $\mathrm{N}$ terminus and regulatory domain ( $R$ domain) $(25,26)$. Residues $46-63$ of the CFTR $N$ terminus are predicted to adopt an $\alpha$-helical conformation that is able to interact with the $\mathrm{R}$ domain. Disruption of the $\mathrm{N}$ terminus- $\mathrm{R}$ domain interaction decreases channel gating and may provide the mechanism by which syntaxins inhibit CFTR channel activity (25-27).

To identify additional regions of the CFTR $N$ terminus important for channel function, we focused on the extreme $\mathrm{N}$-terminal residues (amino acids 1-25), as they are highly conserved across species but to our knowledge have no known function (Figure 1A). Furthermore, several disease-causing missense mutations in this region have been identified in CF patients, which suggests an important functional role for this region of the $\mathrm{N}$ terminus. While characterizing these CFTR mutations, we discovered a proteinprotein interaction between CFTR and filamin-A (FLN-A) that was disrupted by the S13F mutation. FLNs are actin-binding proteins 
A

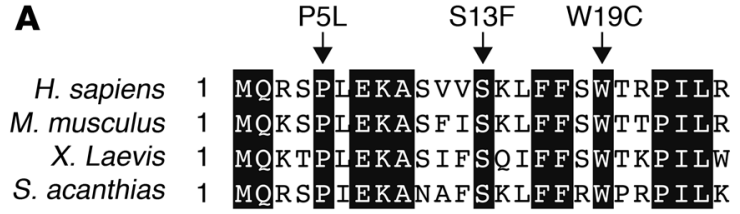

B

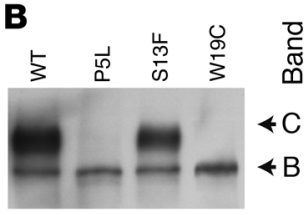

C

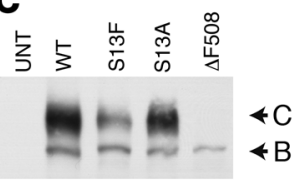

16HBE140-

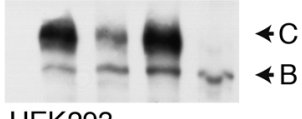

HEK293

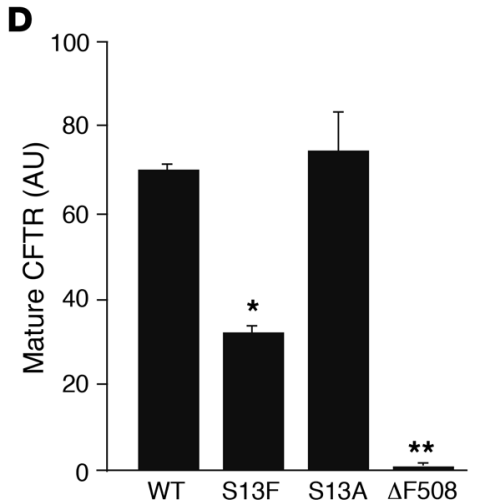

composed of 2 calponin homology domains and 24 Ig-like FLN repeats, the most C-terminal of which mediates dimerization (28). Initially identified as proteins able to crosslink actin filaments to form orthogonal networks (29), FLNs are now known to bind ion channels, receptors, and soluble signaling molecules (30). By forming a direct link to the underlying actin cytoskeleton, FLNs regulate the surface stability, membrane trafficking, and activity of binding partners. Here we demonstrate that FLN-A regulated the metabolic and plasma membrane stability of CFTR. In the absence of FLN-A binding, CFTR was rapidly cleared from the cell surface and degraded in the lysosomes. Our data highlight what we believe to be a novel role for the CFTR $\mathrm{N}$ terminus and provide insights into the molecular mechanism underlying the defect associated with the disease-causing S13F mutation.

\section{Results}

Conserved $N$-terminal residues are required for the biosynthetic maturation of CFTR. Using the CF mutations database (http://www.genet.sickkids.on.ca/cftr), we identified 3 previously described missense mutations in the extreme $\mathrm{N}$ terminus of CFTR: proline 5 to leucine (P5L), serine 13 to phenylalanine $(\mathrm{S} 13 \mathrm{~F})$, and tryptophan 19 to cysteine (W19C) $(31,32)$. To determine whether these mutations would provide insights into the functional roles of the

\section{Figure 2}

The S13F mutation decreases the half-life of CFTR. (A) The indicated CFTR proteins expressed in HEK293 cells were metabolically labeled and incubated in nonradioactive media for the indicated times. CFTR was immunoprecipitated and analyzed by SDS-PAGE/phosphorimager analysis. (B and C) Data were quantitated using ImageQuant software to examine CFTR maturation (B) and degradation (C). For each CFTR protein, data are expressed as percent of control (time 0 for $\mathbf{B}$; band $\mathrm{C}$ at 4 hours for $\mathbf{C}$ ). ${ }^{*} P<0.05$ versus WT CFTR. $n=4$.

\section{Figure 1}

Analysis of N-terminal CFTR mutations. (A) Comparison of amino acids 1-25 of human CFTR with those of other species. Residues conserved in all species are shaded black. Disease-causing missense mutations in CFTR identified from the CF mutation database are indicated above. (B and C) The indicated CFTR proteins were expressed in HEK293 or $16 \mathrm{HBE} 140$ - cells and analyzed by Western blot. Untransfected cells (UNT) served as negative controls. (D) Data from HEK293 cells in $\mathbf{C}$ was quantitated using densitometry. For all CFTR proteins, the level of mature band C CFTR was normalized to that of band B CFTR. ${ }^{\star} P<0.01,{ }^{\star \star} P<0.001$ versus WT CFTR. $n=3$.

CFTR N terminus, we initially expressed P5L, S13F, and W19C CFTR in HEK293 cells and analyzed the proteins by Western blot. The core-glycosylated, ER form of CFTR (band B) migrates with an apparent molecular weight of $160 \mathrm{kDa}$. As CFTR traffics through the cis- and medial-Golgi, the $\mathrm{N}$-glycan is processed to a complex oligosaccharide, decreasing the electrophoretic mobility to $180 \mathrm{kDa}$ (band C) (33). Thus, monitoring the glycosylation state of CFTR provides a useful method to study CFTR maturation and trafficking. Consistent with the results of others (33), WT CFTR was predominantly present as the maturely glycosylated band $\mathrm{C}$ at steady state (Figure 1B). Conversely, P5L and W19C were found only as the ER, core-glycosylated band B protein (Figure 1B). This is not surprising given the large number of CFTR mutations, including the most common $\Delta$ F508 mutation, which result in biosynthetic defects characterized by ER retention and accelerated degradation (33-35). Interestingly, however, S13F CFTR exhibited a clear pool of band C protein (Figure 1, B and $\mathrm{C}$ ) with a significant 2 .2-fold reduction in the band $\mathrm{B} /$ band $\mathrm{C}$ ratio relative to WT CFTR (Figure $1 \mathrm{D}$ ). By contrast, the mutation of serine 13 to another amino acid, alanine, had no effect on the band $\mathrm{B} /$ band $\mathrm{C}$ ratio relative to WT CFTR (Figure 1, C and $\mathrm{D})$. We observed similar decreases in the band $\mathrm{C}$ protein for $\mathrm{S} 13 \mathrm{~F}$ CFTR in an airway epithelial cell line, 16HBE140-, which demonstrates that the defect in S13F CFTR maturation is not cell type specific and is recapitulated in airway epithelial cells (Figure 1C). Based on the band $\mathrm{B} /$ band $\mathrm{C}$ ratio, the S13F mutation appeared to be distinct from the majority of CFTR mutations identified to date. Therefore, we sought to characterize the molecular defect associated with this mutation.

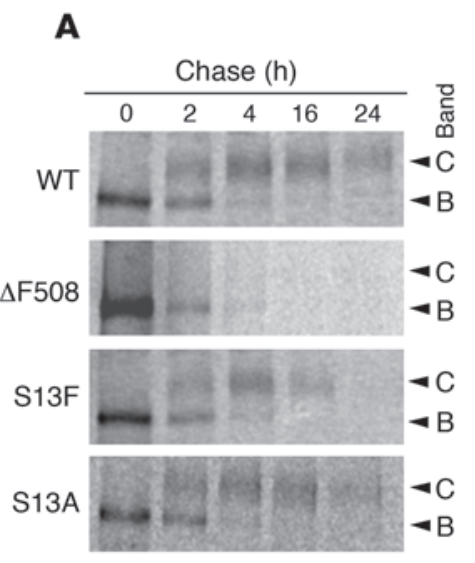

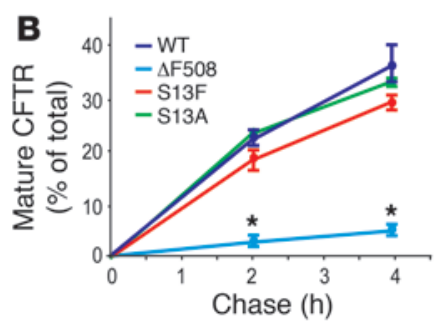

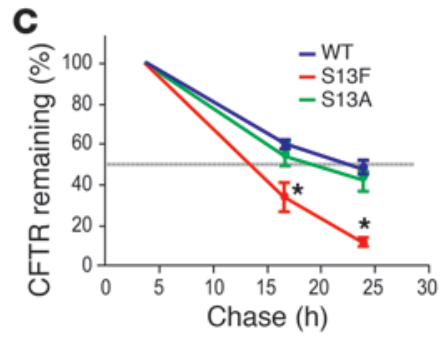



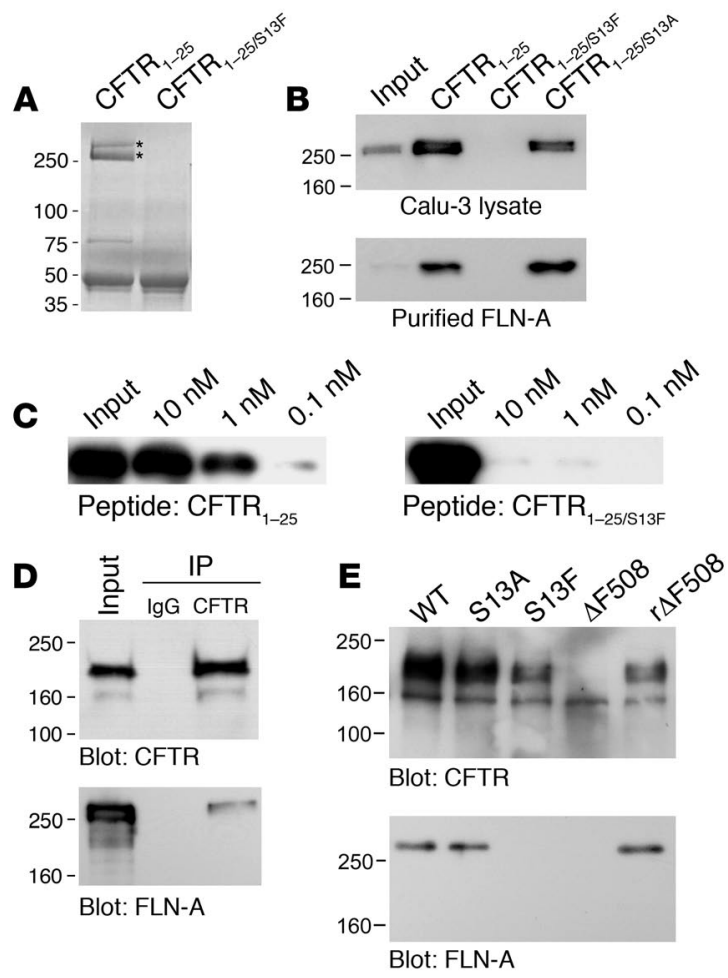

The S13F mutation decreases the half-life of CFTR. The decrease in the band $\mathrm{B} /$ band $\mathrm{C}$ ratio observed for S13F CFTR reflects either a defect in CFTR maturation and/or an increase in the degradation of the mature protein. Therefore, we monitored the maturation and degradation in metabolic pulse-chase experiments over a 24-hour period. WT, S13F, and S13A CFTRs were transiently expressed in HEK293 cells, labeled, immunoprecipitated, and analyzed by autoradiography (Figure 2A). As an additional control, we included $\Delta$ F508 CFTR in these studies because this mutant represents a biosynthetically defective CFTR protein that fails to exit the ER. After 4 hours of chase, WT and S13A CFTRs were present almost exclusively as the mature band $\mathrm{C}$, while no band C was observed for $\triangle F 508$ (Figure 2B). Like WT and S13A CFTR, S13F CFTR was clearly processed from the band B to band C protein during the first 4 hours of chase. These data indicate that the major defect associated with the S13F mutation does not involve protein folding or ER exit.

We next measured the rate of degradation of mature band $\mathrm{C}$ CFTR proteins by comparing the CFTR present after 4, 16, and 24 hours of chase. The half-life was similar between WT and S13A CFTR (22.5 and 22.6 hours, respectively; Figure 2C). However, the half-life of S13F CFTR was reduced by more than 50\% (10.8 hours; $P<0.05)$. Taken together, the results of our metabolic pulse-chase studies demonstrate that the S13F mutation decreases the stability of the mature band C CFTR.

FLNs directly associate with the CFTR $N$ terminus. We hypothesized that the $\mathrm{N}$ terminus of CFTR engages in protein-protein interactions that regulate its stability, which may be affected by the S13F mutation. To test our hypothesis, we used affinity chromatography to purify proteins associated with the CFTR N terminus. CFTR peptides corresponding to residues $1-25$ of either WT CFTR $\left(\mathrm{CFTR}_{1-25}\right)$ or S13F CFTR (CFTR $1-25 / \mathrm{S} 13 \mathrm{~F}$ ) were used as affinity ligands to purify CFTR interacting proteins from Calu-3 airway epithelial cell lysates.

\section{Figure 3}

FLNs interact with the CFTR N terminus. (A) Coomassie-stained gel of proteins that copurified with CFTR ${ }_{1-25}$ or CFTR $_{1-25 / \mathrm{S} 13 \mathrm{~F}}$ from Calu-3 cell lysates. Bands identified as FLN-A and FLN-B are indicated by an asterisk. (B and $\mathbf{C}$ ) FLN-A Western blots of CFTR peptide pulldowns from (B) Calu-3 lysates $(100 \mu \mathrm{g})$ or (C) purified FLN-A (500 nM). Inputs are $5 \%$ of the total. S13A, CFTR 1 -25/S13A. (D) Endogenous CFTR was immunoprecipitated from solubilized Calu-3 membranes and analyzed by Western blot for CFTR or FLN-A. (E) The indicated CFTR proteins were expressed in HEK293 cells at $37^{\circ} \mathrm{C}$ or $28^{\circ} \mathrm{C}$ to temperature-rescue $\Delta$ F508 ( $r$ F508). CFTR was immunoprecipitated, and samples were analyzed by Western blot for CFTR or FLN-A. Protein molecular weights in $\mathrm{kDa}$ are shown to the left of the blots.

Bound proteins were eluted, separated by SDS-PAGE, and visualized by Coomassie staining. We observe several nonspecific bands associated with both $\mathrm{CFTR}_{1-25}$ and $\mathrm{CFTR}_{1-25 / \mathrm{S} 13 \mathrm{~F}}$. However, 2 high-molecular weight bands specifically copurified with the $\mathrm{CFTR}_{1-25}$ but not the $\mathrm{CFTR}_{1-25 / \mathrm{S} 13 \mathrm{~F}}$ peptides (Figure $3 \mathrm{~A}$ ). These bands were excised, digested in-gel with trypsin, and analyzed by matrix-assisted laser desorption/ionization tandem mass spectrometry (MALDI-MS/ MS). The bands were confidently identified as FLN-A and FLN-B (also referred to as ABP280 and ABP278, respectively), with sequence coverages of 34\% for FLN-A and 47\% for FLN-B.

Consistent with the MS data, FLN-A specifically copurified from Calu-3 cell lysates with the $\mathrm{CFTR}_{1-25}$ peptide as assessed by Western blot (Figure 3B). Moreover, purified, full-length FLN-A interacted with $\mathrm{CFTR}_{1-25}$, demonstrating that the interaction was both direct (Figure 3B) and concentration dependent (Figure 3C). The incorporation of the S13F mutation into the $\mathrm{CFTR}_{1-25}$ peptides nearly abolished the interaction with FLN-A in these assays.

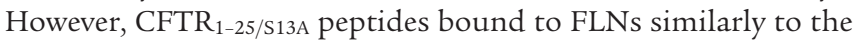
CFTR $_{1-25}$ peptides (Figure 3B). Taken together, these data demonstrate that WT CFTR can directly interact with FLNs and that the S13F mutation disrupts this interaction. Thus, the reduction relative to WT CFTR in the band $\mathrm{B} /$ band $\mathrm{C}$ ratio observed for the $\mathrm{S} 13 \mathrm{~F}$ mutation, but not the S13A mutation, correlates with the ability to bind FLN-A in vitro.

CFTR and FLN-A associate in vivo. In order to associate within a biological context, CFTR and FLN-A must be coexpressed in the same cells and at least partially colocalize to the same subcellular compartment. Therefore, we used immunoprecipitation to determine whether CFTR and FLN-A colocalize in relevant tissues. FLN-A coimmunoprecipitated with endogenous CFTR from Calu-3 cell lysates, but not with an IgG control (Figure 3D). Furthermore, FLN-A coprecipitated with WT and S13A CFTR expressed in HEK293 cells, but not with S13F CFTR (Figure 3E). Thus, the S13F mutation disrupted FLN-A binding to CFTR in vivo. Additionally, we found that ER-retained $\triangle F 508$ CFTR did not interact with FLN-A. Based on these data and the results of our pulse-chase studies, we speculated that FLNs do not interact with CFTR in the ER. To test this hypothesis, HEK293 cells expressing $\triangle \mathrm{F} 508 \mathrm{CFTR}$ were grown at $28^{\circ} \mathrm{C}$. At this permissive temperature, we found that $\Delta \mathrm{F} 508 \mathrm{CFTR}$ partially matured, as indicated by the appearance of band C (Figure 3E). As shown by immunoprecipitation, FLN-A copurified with rescued $\Delta \mathrm{F} 508$ CFTR, but not with $\Delta$ F508 CFTR from cells grown at $37^{\circ} \mathrm{C}$. Thus, we concluded that CFTR and FLN-A interact in a post-ER compartment. Importantly, the S13F mutation, which disrupted FLN binding in pulldown and immunoprecipitation assays, provided 
A

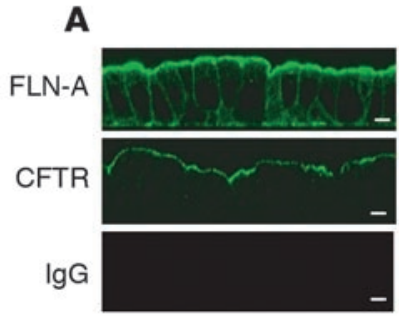

B

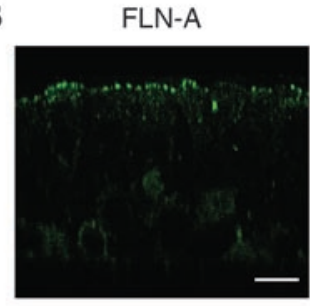

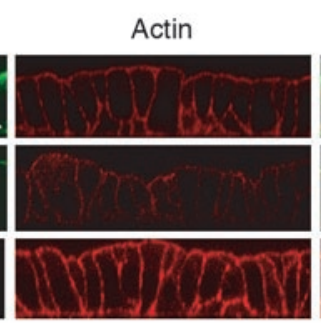

Actin + DAPI

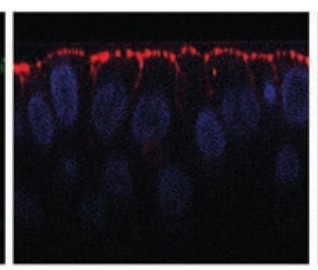

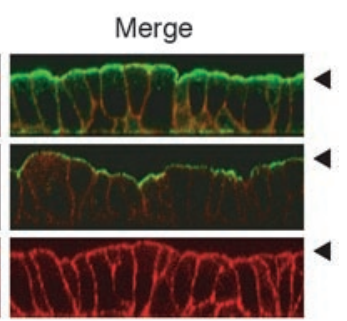

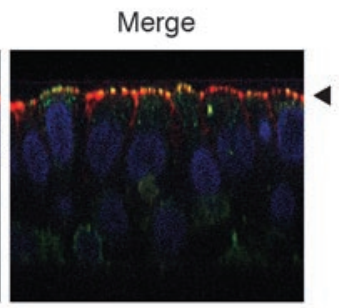

Figure 4

FLN-A localizes to the subapical membrane of airway epithelia. FLN-A localization was analyzed by immunofluorescence in (A) Calu- 3 cells or (B) primary cultures of WD-HBEs as described in Methods. Arrowheads indicate the apical membrane. CFTR staining and purified, whole mouse IgG as a negative control are also shown in Calu-3 cells. Scale bars: $10 \mu \mathrm{m}$.

cytic trafficking of their transmembrane binding partners (36-42). To determine whether FLNs regulate the plasma membrane pool of CFTR, we used CFTR proteins with an extracellular HA tag. The surface pool of CFTR was detected by immunofluorescence in unpermeabilized baby hamster kidney (BHK) cells expressing either WT, S13A, S13F, or $\triangle \mathrm{F} 508$ HA-CFTR. We observed significant amounts of WT and S13A CFTR at the cell surface, while no signal

us with a powerful tool to study the functional significance of this interaction in cells.

In polarized Calu-3 cells and primary cultures of well differentiated human bronchial epithelia (WD-HBE), FLN-A was associated with intracellular vesicular structures and the cell cortex, where it was highly concentrated at the apical compartment (Figure 4, A and B). Likewise, CFTR was observed primarily at the apical cell surface in Calu-3 cells at steady state. The strong apical localization of FLN-A in polarized epithelia suggest that CFTR and FLN-A may interact at or near the cell surface; however, these results do not preclude an interaction in an intracellular compartment.

CFTR surface expression is regulated by FLNs. Previous studies have shown that FLNs can regulate the cell surface expression and endo- was observed for $\Delta \mathrm{F} 508$ (Figure $5 \mathrm{~A}$ ). In permeabilized cells, $\Delta \mathrm{F} 508$ displayed a reticular staining pattern (Figure $5 \mathrm{~A}$ ) that colocalized with ER markers (data not shown), consistent with the intracellular retention of this protein. Unlike $\Delta$ F508 CFTR, we observed surface staining for S13F CFTR; however, the staining was greatly reduced compared with WT and S13A CFTR. We quantitatively analyzed these differences using cell surface ELISAs to measure the plasma membrane pool of CFTR relative to the total cellular CFTR. In agreement with our observations by immunofluorescence, a substantial pool of WT and S13A CFTR resided on the cell surface (41.2\% and $37.8 \%$, respectively), while no $\Delta \mathrm{F} 508$ was observed (Figure $5 \mathrm{~B}$ ). Furthermore, the surface pool of S13F CFTR was significantly less than that of WT and S13F CFTR $(8.7 \% ; P<0.01)$ but significantly greater
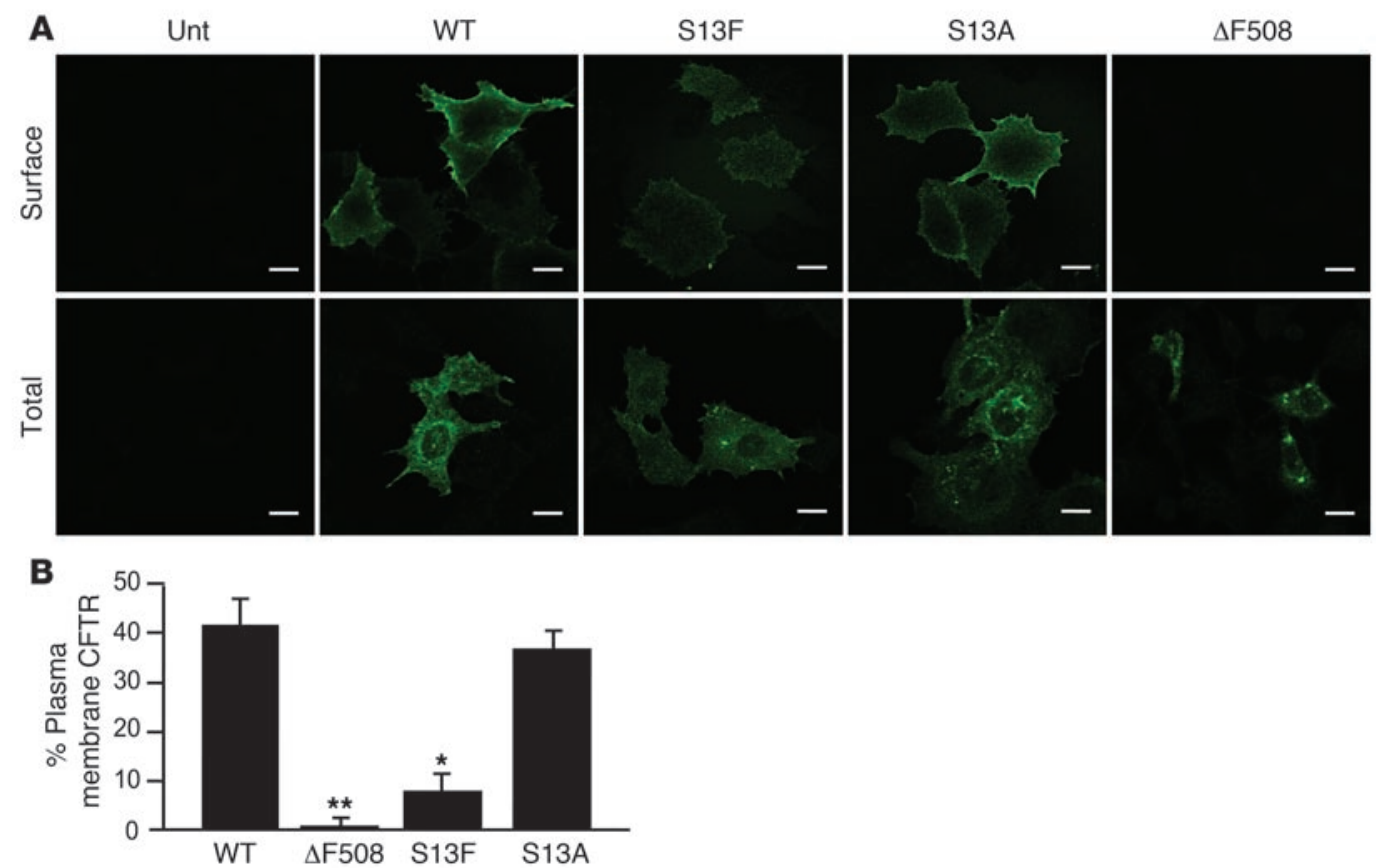

Figure 5

Surface expression of S13F CFTR is decreased. The indicated HA-CFTR proteins were expressed in BHK cells. CFTR proteins were analyzed by (A) immunofluorescence or (B) surface ELISA in unpermeabilized cells (surface pool) or detergent permeabilized cells (total CFTR) using HA antibodies as described in Methods. Scale bars: $10 \mu \mathrm{m} .{ }^{*} P<0.05,{ }^{* \star} P<0.005$ versus WT CFTR. $n=36$. 
A
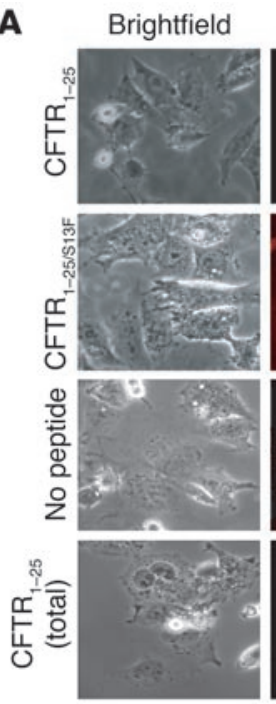

C
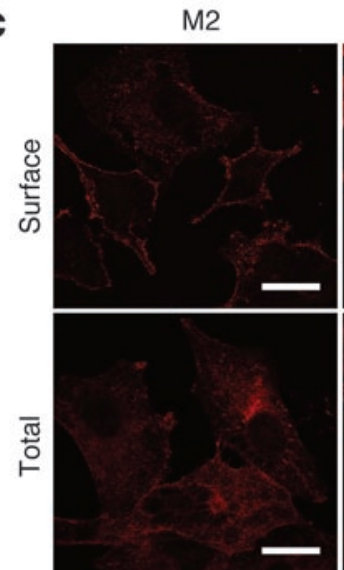

CFTR
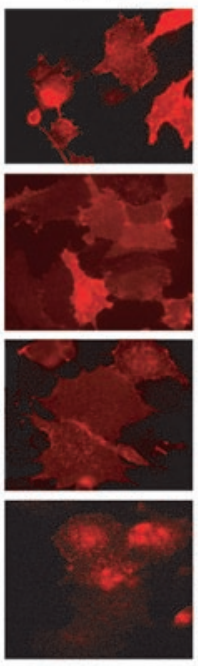

Probe
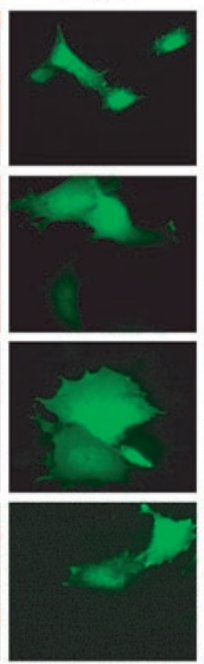

A7

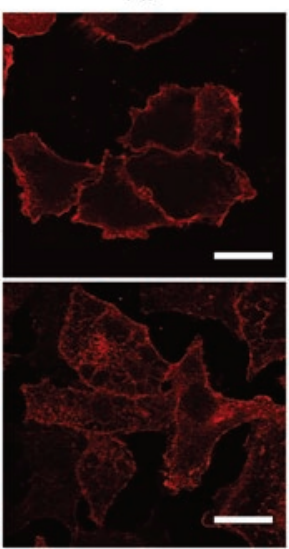

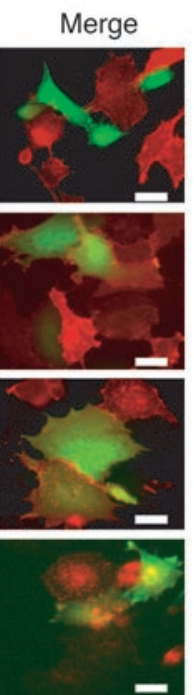

D
B

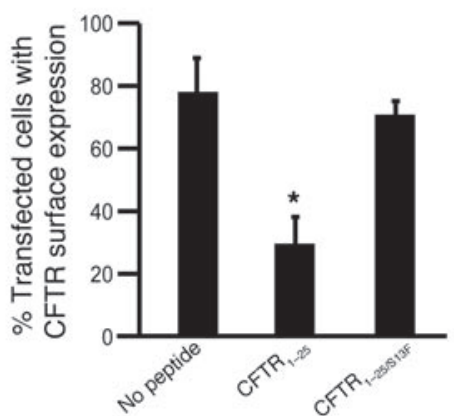

Surface expression

$13.7 \%$

$41.5 \%$

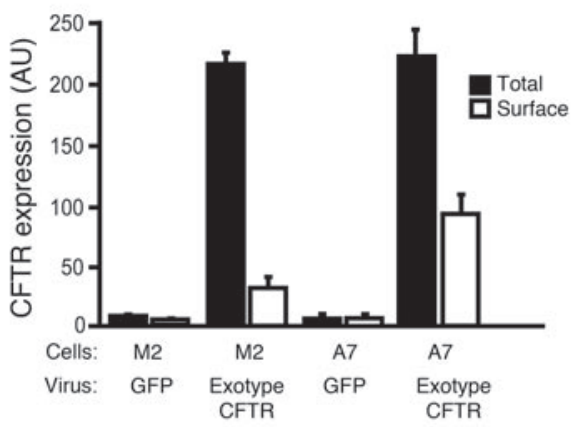

Figure 6

Perturbations in FLN-A binding decrease the surface expression of WT CFTR. (A) The indicated CFTR peptides were introduced into BHK cells stably expressing WT HA-CFTR using the Pro-Ject delivery system. Cell surface CFTR was assessed by labeling unpermeabilized cells with HA antibodies followed by Alexa Fluor 594-labeled secondary antibodies. Transfected cells were identified by the uptake of fluorescently labeled $F\left(a b^{\prime}\right)$ fragments included in the transfection complexes. (B) Peptide-transfected cells were assessed for CFTR surface expression as described in Methods. ${ }^{*} P<0.05 . n=4$ with 50 transfected cells counted per individual experiment. (C and D) HA-CFTR or GFP was expressed using adenovirus in FLN-A-replete cells (M2) or FLN-A-reexpressing cells (A7). The surface expression of CFTR was analyzed by immunofluorescence (C) and surface ELISA (D) as described in Figure 5. Scale bars: $10 \mu \mathrm{m}$.

than that of $\triangle$ F508 CFTR $(P<0.05)$. Thus, in the absence of FLN binding, S13F CFTR localizes to the cell surface, albeit at reduced levels compared with WT CFTR.

The S13F mutation reflects a loss of FLN binding. We used a cationic lipid transfection system to introduce CFTR peptides into cells and disrupt the CFTR-FLN interaction in situ. Using this approach, we successfully delivered several fluorescent probes into cells such as FITC-labeled antibodies, $\mathrm{F}\left(\mathrm{ab}^{\prime}\right)$ fragments, and streptavidin. While these probes were abundantly delivered to cells, the fraction of transfected cells was low $(<10 \%)$. The low transfection efficiency precluded us from using assays such as cell surface ELISA in these experiments. Therefore, we analyzed the effects of the peptide transfection using single cell assays. We examined the surface expression of CFTR in BHK cells stably expressing HA-CFTR that were transfected with a nonbiotinylated CFTR $\mathrm{C}_{1-25}$ peptide cotransfected with FITC-labeled $\mathrm{F}\left(\mathrm{ab}^{\prime}\right)$ fragments as a transfection mark- er. We found that the transfected cells exhibited little to no CFTR surface expression, in contrast to untransfected cells (Figure 6, A and B). Importantly, this effect was not observed when the S13F peptide or the $\mathrm{F}\left(\mathrm{ab}^{\prime}\right)$ fragments alone were transfected.

In addition, we analyzed the plasma membrane pool of CFTR in the well characterized melanoma cell lines M2 (which lacks endogenous FLN-A) and A7 (which stably reexpresses FLN-A). In agreement with our earlier experiments, the surface expression of WT CFTR was clearly reduced in M2 cells compared with A7 cells as assessed by immunofluorescence (Figure 6C). As determined by ELISA, the fraction of WT CFTR on the plasma membrane was also significantly lower in M2 cells than in A7 cells (13.7\% and $41.5 \%$, respectively; Figure $6 \mathrm{D}$ ). Thus, using several different approaches to manipulate the CFTR-FLN interaction, we consistently found that FLN binding is required for the normal surface expression of CFTR. 
WT CFTR
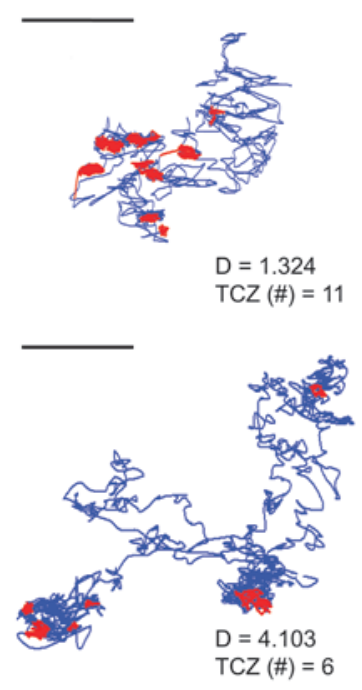

S13F CFTR
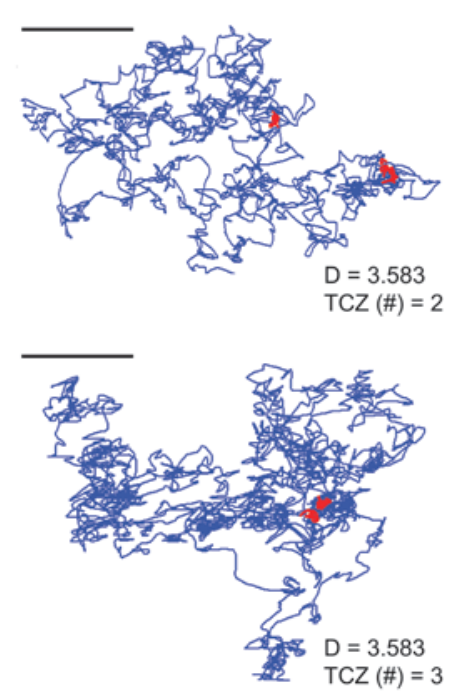

FLNs regulate membrane compartmentalization. We next used single particle tracking (SPT) experiments to further analyze the effects of FLN binding on plasma membrane CFTR. SPT allowed us to track plasma membrane CFTR at the molecular level with video time resolution $(30 \mathrm{~Hz})$. Previous studies have used this technique to observe the effects of lipid raft compartmentalization, membrane confinement, and cytoskeletal anchorage (43-47). We reasoned that SPT would allow us to observe changes in the membrane dynamics of CFTR associated with FLN-mediated cytoskeletal anchorage. Cell surface HA-CFTR proteins were labeled in live HeLa cells using biotinylated anti-HA antibodies followed by colloidal gold-conjugated anti-biotin antibodies. We then tracked the trajectories of single gold particles using computer-enhanced video microscopy. Trajectories of at least 50 different gold particles were analyzed from cells expressing WT or S13F CFTR. WT CFTR diffused randomly across the plasma membrane, followed by short periods of transient confinement (Figure 7). On average, WT CFTR spent $8.41 \%$ of the total recording time in transient confinement zones (TCZs) ranging 100-300 nm in diameter. To determine whether the association with FLNs is important for the transient confinement of CFTR, we examined S13F CFTR by SPT. We found that S13F CFTR exhibited significantly less transient confinement than WT CFTR (Figure 7). The confinement of S13F CFTR was reduced by more than $50 \%$ relative to $\mathrm{WT}$, which reflects a decrease in both the number of confinements and the time spent in a single TCZ (Table 1). Interestingly, the diffusion coefficients of WT and S13F were not greatly different (3.45 \pm 0.41 versus $2.73 \pm 0.71 \times 10^{-10} \mathrm{~cm}^{2} / \mathrm{s}$ ), suggesting that for CFTR, incorporation into TCZ does not alter the rate of diffusion in the membrane (Table 1). Taken together, the results of our surface labeling and SPT experiments demonstrate that FLNs modulate both the expression and the confinement of CFTR at the plasma membrane. Furthermore, these data support our hypothesis that FLNs play a role in the cell surface stability and spatial organization of CFTR by coupling the channel to the actin cytoskeleton.

$S 13 F C F T R$ is rapidly cleared from the cell surface. At the plasma membrane, CFTR is rapidly internalized into clathrin-coated vesicles and efficiently returned to the cell surface via endocytic recycling $(13,48-52)$. During the SPT experiments, we qualitatively observed

\section{Figure 7}

The membrane dynamics of S13F CFTR is altered. WT and S13F CFTRs were analyzed by SPT in HeLa cells. Blue lines show trajectory of individual CFTR proteins labeled with gold particles during a 60 -second recording. Red areas show regions of transient confinement. The diffusion coefficient (D) and number of TCZs in each CFTR are also indicated. Scale bars: $1 \mu$ M. $n=45$.

many more S13F proteins internalized compared with WT CFTR. Although these proteins were not analyzed by SPT, this result prompted us to ask whether FLN-A binding affects the internalization rate of CFTR using a modified surface ELISA. HeLa cells transiently expressing either WT or S13F HA-CFTR were chilled to $4{ }^{\circ} \mathrm{C}$ to block internalization and labeled with anti-HA antibodies. After removing excess antibodies, the cells were warmed to $37^{\circ} \mathrm{C}$ for $0,5,10$, and 15 minutes and then fixed, labeled with IRDyelabeled secondary antibodies, and analyzed using a LI-COR Biosciences Odyssey instrument. Because the cells were not permeabilized, this approach allowed us to follow the loss of the surface pool due to internalization over time. We found that the surface expression of S13F decreased significantly more rapidly than that of WT CFTR (Figure 8). At the 5-minute time point, significantly more S13F CFTR was internalized compared with WT CFTR (25.4\% versus $13.8 \% ; P>0.05)$. This trend was more pronounced at the 15 -minute time point, where $61.5 \%$ of S13F CFTR was cleared from the cell surface compared with $25.1 \%$ for WT CFTR. Thus, the FLN-binding mutant S13F CFTR is less stable on the cell surface. Importantly, the differences observed between WT and S13F CFTR at the 5-minute time point likely reflect changes in internalization. However, CFTR internalization did not change between the 10- and 15-minute time points for WT CFTR, indicating that recycling was occurring. Conversely, cell surface S13F CFTR continued to be lost between the 10- and 15-minute time points, consistent with defects in recycling kinetics.

The accelerated degradation of S13F CFTR is primarily mediated by lysosomes. We next tested whether the endocytic trafficking of CFTR was regulated by FLNs. HeLa cells transiently expressing either WT or S13F HA-CFTR were chilled to $4^{\circ} \mathrm{C}$ to block internalization and labeled with anti-HA antibodies. After removing excess antibodies, the cells were warmed to $37^{\circ} \mathrm{C}$ for 2 , 4 , or 8 hours and then fixed, permeabilized, and labeled with fluorescent secondary antibodies. Using the same HA-CFTR construct stably expressed in BHK cells, Gentzsch et al. found that internalized CFTR is localized with markers to early endosomes by 1 to 2 hours, late endosomes by 4 hours, and lysosomes by 48 hours (53). In our experiments, both WT and S13F CFTR exhibited partial overlap with the early endosome marker EEA1 and internalized transferrin at the 2-hour time point (data not shown). However, we observed striking dif-

\section{Table 1}

SPT data quantitation for WT and S13F CFTR proteins

$\begin{array}{lccc}\text { CFTR } & \begin{array}{c}\text { Diffusion coefficient } \\ \left(\times \mathbf{1 0}^{-\mathbf{1 0}} \mathbf{c m}^{2} / \mathbf{s}\right)\end{array} & \begin{array}{c}\text { Relative confinement } \\ \text { time }(\%)\end{array} & \begin{array}{c}\text { TCZ dwell } \\ \text { time }(\mathbf{s})\end{array} \\ \text { WT } & 3.45 \pm 0.41 & 8.41 \pm 2.50 & 1.00 \pm 0.32 \\ \text { S13F } & 2.73 \pm 0.71 & 4.45 \pm 1.16^{\mathrm{A}} & 0.30 \pm 0.03^{\mathrm{A}}\end{array}$

Values are mean \pm SEM quantified from data in Figure 7. $n=45$. AP $<0.001$. 


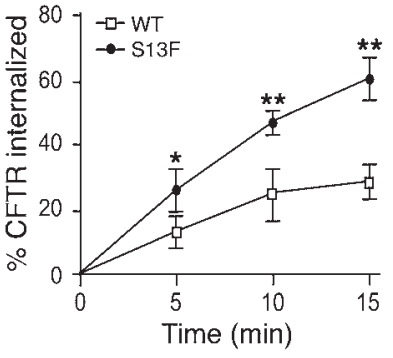

ferences between WT and S13F CFTR at the 24-hour time points (Figure 9A). Consistent with the findings of Gentzsch et al. (53), we observed little colocalization of WT CFTR with lysosomes by 24 hours. In contrast, we found some colocalization between S13F CFTR and lysosomes by 8 hours, which was more evident by 24 hours (Figure 9A).

In our initial studies of the CFTR N-terminal mutations, we found that the steady-state distribution of S13F CFTR displayed

\section{Figure 8}

S13F CFTR is internalized more rapidly than is WT CFTR. WT or S13F CFTRs were transiently expressed in BHK cells. The cell surface pool was labeled with HA antibodies as described in Methods. Antibodylabeled CFTR proteins were allowed to internalize for the indicated times, and the disappearance of plasma membrane CFTR was monitored. ${ }^{*} P<0.05,{ }^{*} P<0.005$ versus WT CFTR. $n=3$ with 24 replicates per experiment for each condition.
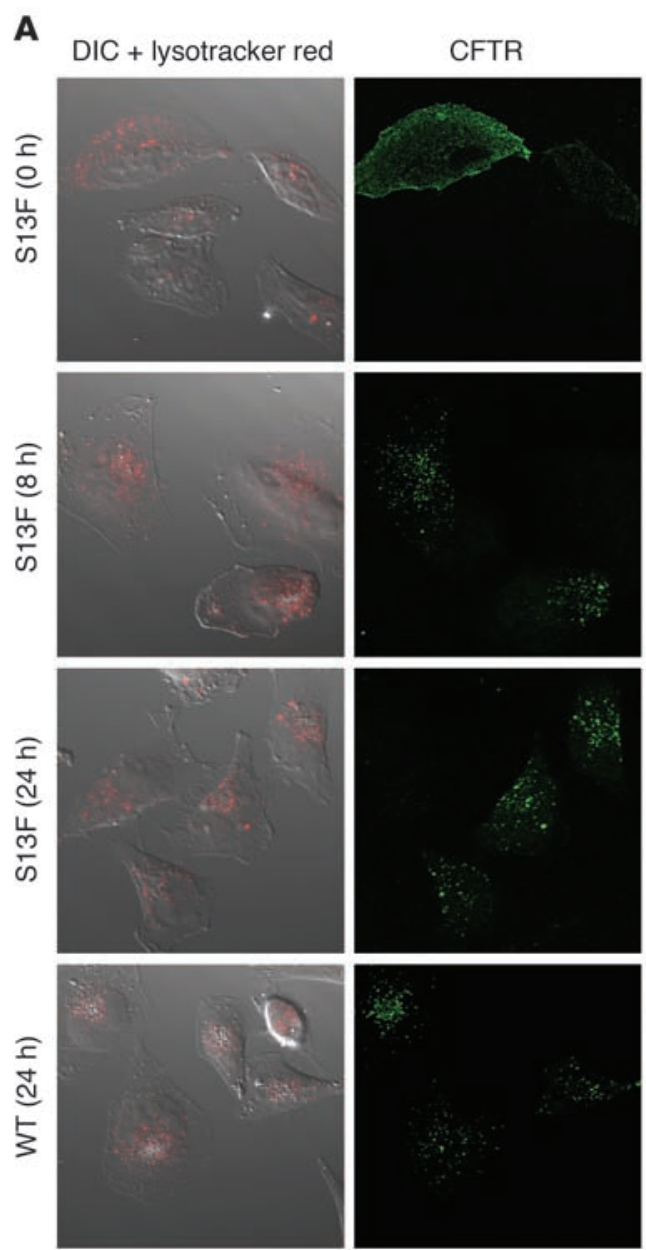

a decrease in the mature band $\mathrm{C}$ protein, which reflects increased degradation. Additionally, S13F was prematurely sorted to lysosomes, which may explain why mature S13F CFTR was degraded more rapidly than the WT protein. Therefore, we examined the half-life of WT, S13F, and S13A CFTR by pulse chase in the presence of the lysosomal protease inhibitor leupeptin. Strikingly, leupeptin significantly increased the half-life of S13F CFTR from 13.4 hours to 18.3 hours (Figure 9, B and C). Although leupeptin did
CFTR + lysotracker red
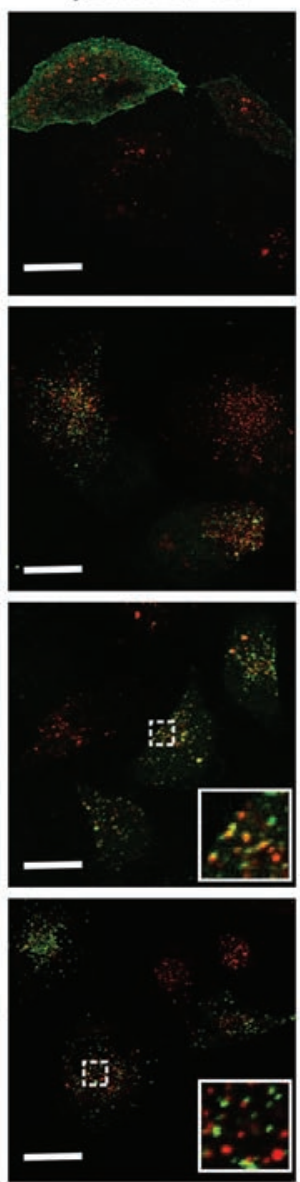

B

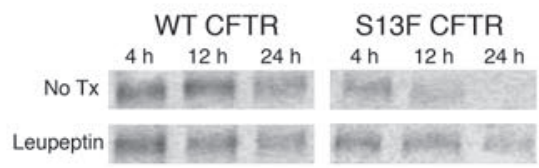

C

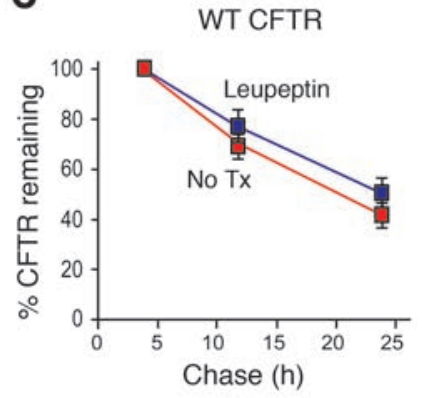

S13F CFTR

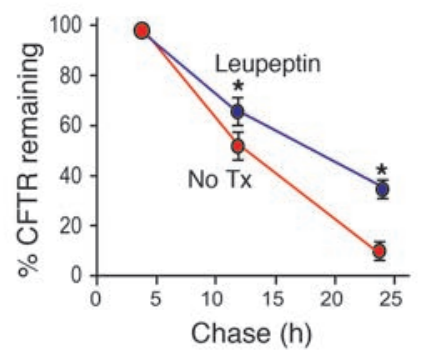

\section{Figure 9}

S13F CFTR prematurely accumulates in the lysosomes, where it is degraded. (A) HA-CFTR proteins were expressed in BHK cells. The surface pool of CFTR was labeled with HA antibodies and allowed to internalize for the indicated times. CFTR was visualized using Alexa Fluor 488 antibodies, and lysosomes were labeled with lysotracker red. Both immunofluorescent and differential interference contrast (DIC) microscopy images are shown. Insets show colocalization of CFTR and the lysosomal marker. Magnification, $\times 63$; insets, $\times 189$. (B) WT CFTR and S13F CFTRs were analyzed by metabolic labeling in pulse-chase experiments in the presence of lysosomal protease inhibitors (Leupeptin) or with no treatment (No tx). Representative gels are shown for WT CFTR and S13F CFTR. (C) Quantitation of the data in B. ${ }^{*} P<0.05$ versus control. $n=3$. 
not fully rescue S13F to the 21.9-hour half-life of WT CFTR (Figure 9, B and C), it suggests that lysosomally mediated degradation accounts for the majority of S13F turnover.

\section{Discussion}

Here we report the characterization of what we believe to be a novel protein-protein interaction between the $\mathrm{N}$ terminus of CFTR and FLN proteins that is required for the surface expression and stability of CFTR. In addition, we found that the S13F mutation in CFTR disrupted the interaction with FLNs, resulting in a decrease in both metabolic and plasma membrane stability of CFTR. To our knowledge, the disease-causing S13F mutation is the first missense mutation in CFTR found to disrupt a protein-protein interaction.

FLNs are best appreciated as structural proteins that regulate cellular architecture. However, FLNs are now known to directly interact with membrane-spanning proteins including ion channels, adhesion molecules, and $G$ protein-coupled receptors $(36-42,54)$. Based on these studies, it is clear that FLNs stabilize their binding partners at the cell surface. For example, the plasma membrane levels of the glycoprotein Ib $\alpha$ (GPIb $\alpha$; ref. 37), the D2and D3-dopamine receptors (D2R and D3R, respectively; ref. 38), the inwardly rectifying potassium channel 2.1 (Kir2.1; ref. 40), furin (39), and the calcium-sensing receptor (CaR; ref. 42) are all significantly reduced in the absence of FLN-A binding. Likewise, we found an approximately 5 -fold reduction in the cell surface pool of S13F CFTR relative to WT CFTR. This effect is specific to a loss of FLN binding, because decreased surface expression was observed for WT CFTR in the presence of a competitive peptide inhibitor or when expressed in cells lacking FLN-A (M2 cells). The decrease in the plasma membrane CFTR was greater for S13F in multiple cell types than for WT CFTR in the M2 cells. We hypothesize that this difference can be accounted for by the fact that the S13F mutation disrupted the interaction with both FLN-A and FLN-B, whereas the M2 cells expressed FLN-B, which may partially compensate for the loss of FLN-A.

The decrease in CFTR surface expression coincides with altered plasma membrane mobility and increased endocytosis. Consistent with the findings of Bates et al. (55), we found that CFTR was highly mobile on the cell surface, where it underwent periods of diffusion followed by brief transient confinements. For S13F CFTR, the partitioning of CFTR into confinement zones was significantly reduced (approximately 50\%). Previous studies have shown that transient confinement may represent either cytoskeletal anchorage and/or partitioning into lipid microdomains (56). However, because cholesterol depletion has a minor effect on CFTR transient confinement (47), we favor the hypothesis that FLN is involved in either directly tethering CFTR to the cytoskeleton and/or creating a cytoskeletal meshwork that confines goldlabeled CFTR by steric interactions with its cytoplasmic domain. Studies from our lab and others have shown that the $\mathrm{C}$ terminus of CFTR also interacts with the cytoskeleton via sodium-hydrogen exchanger regulatory factor (NHERF) proteins and ezrin (57, 58 ). We predict that cytoskeletal interactions with the CFTR C terminus likely account for the residual membrane confinements observed for S13F CFTR. In addition, we observed increased endocytosis of S13F CFTR from the plasma membrane relative to the WT protein. This result is consistent with the findings for other FLN-binding proteins such as furin (39), the calcitonin receptor (41), and the prostate-specific membrane antigen (PSMA; ref. 36) that display accelerated internalization in cells lacking FLN-A.
Although the mechanism is not clear, it is exciting to speculate that the transient confinement of CFTR in the plasma membrane delays its incorporation into endocytic vesicles.

We also found that the half-life of S13F was decreased compared with WT CFTR, suggesting a role for FLNs in the metabolic stability of CFTR. Unlike $\Delta$ F508, P5L, or W19C, S13F CFTR displayed a clear pool of band $\mathrm{C}$ protein in both heterologous expression systems and epithelial cells. However, this pool was substantially reduced (approximately 50\%) relative to WT CFTR. These results, together with those of our pulse-chase studies, lead us to conclude that a primary defect associated with the S13F mutation is a decrease in the stability of the mature, complex glycoslyated protein. Likewise, the metabolic stability was altered for other FLN-binding proteins when FLN-A is absent or the interaction is disrupted. Feng et al. report that the interaction with FLN-A stabilizes nacent GPIb $\alpha$ in the ER, which is critical for both the metabolic stability and the surface expression of this protein (59). Our findings are not consistent with this mechanism for CFTR, as the metabolic stability was decreased for the mature band $\mathrm{C}$ protein as opposed to band B CFTR. Furthermore, our observation that ER-retained $\Delta$ F508 did not interact with FLN-A as assessed by coimmunoprecipitation, but temperature-rescued $\Delta$ F508 CFTR did, suggests that the CFTR-FLN-A interaction likely takes place in a post-ER compartment.

An important consideration is the relationship between the loss of CFTR plasma membrane stability and the premature degradation of the channel. Previous studies have shown that increasing CFTR internalization or decreasing endocytic recycling do not necessarily affect the metabolic stability of mature CFTR. For example, the R31L or N287Y mutations may introduce a nonnative internalization motif in CFTR and result in increased plasma membrane internalization $(8,9)$. Additionally, the deletion of the C-terminal PDZ binding motif results in decreased apical surface expression, which reflects less efficient recycling and not a change in endocytosis rates (60). However, the metabolic stability of mature CFTR was not different from that of WT in these studies. Sharma et al. demonstrated that CFTR proteins that are misfolded, however, escape the ER quality control (e.g., temperature-rescued $\Delta \mathrm{F} 508$ ), are not stable on the cell surface, are rapidly internalized, and are degraded via the proteasome (61). The accelerated degradation associated with the S13F mutation and loss of FLN binding is distinct from the mechanism proposed for temperature-rescued $\Delta \mathrm{F} 508$ because the degradation of S13F is primarily mediated by the lysosomes. The half-life of S13F CFTR can be restored close to that of WT CFTR by inhibiting lysosomal proteases. In addition, we found that S13F accumulated in a lysosomal compartment much more rapidly than did WT CFTR. It is likely that the lysosomal targeting and degradation of S13F CFTR reflects alterations in endocytic trafficking as a result of the loss of FLN binding. Similarly, the membrane trafficking of other FLN-A-binding proteins including furin (39), PSMA (36), and the calcitonin receptor (41) are altered in FLN-A-null cells, resulting in the mislocalization of these proteins to various endosomal compartments. Thus, the decreased metabolic stability of mature S13F CFTR may reflect both its instability at the cell surface and defective endocytic trafficking.

Although not tested here, the interaction with FLNs may also be important for regulating the channel activity of CFTR at the cell surface. The interaction with FLN-A results in clustering of the Kir2.1 channels and the hyperpolarization-activated cyclic nucleo- 
tide-gated channels (HCN1) at the cell surface, thereby increasing current density $(40,54)$. Additionally, FLNs can organize multiprotein complexes and compartmentalize regulatory factors with receptors and ion channels. Recent studies have shown a role for FLN in the organization of cAMP signaling machinery upstream of CFTR activation including the $\beta_{2}$-adrenergic receptor, $G \alpha_{s}$, and adenylate cyclases (62). Furthermore, electrophysiological studies have shown that in the FLN-null M2 cells, both whole-cell and single-channel CFTR currents are reduced in response to cAMP/ PKA activity (63). While reductions in whole-cell currents in part reflect less plasma membrane CFTR, the decreased openings of single CFTR channels suggest that FLNs directly affect gating. Thus, FLNs may additionally compartmentalize CFTR with relevant signaling molecules.

It is therefore clear that FLNs regulate multiple aspects of CFTR biology. Our observations are consistent with findings in $\mathrm{CF}$ patients, which suggest that $\mathrm{S} 13 \mathrm{~F}$ is a significant disease-causing mutation. The clinical manifestations of CFTR mutations range from mild, resulting in elevated sweat chlorides, to severe, resulting in pulmonary defects and pancreatic insufficiency. In an individual with S13F paired with a known mild mutation, T338I, elevated sweat chloride was the only clinical manifestation (31). However, a patient with the S13F mutation paired with a frame-shift mutation, 2185ins A, displayed symptoms of CF including elevated sweat chloride, Pseudomonas aeruginosa lung infection, and pancreatic insufficiency (32). In preliminary studies, nasal potential difference measurements from the individual with $\mathrm{S} 13 \mathrm{~F} / 2185$ ins $A$ were consistent with a functional loss of CFTR at the cell surface, as little to no CFTR activity was detected (M. Knowles, unpublished observations). However, more individuals with S13F should be examined to confirm the disease severity of this mutation.

CF results from the loss of CFTR from the cell surface of epithelial tissues. Because the most common disease-causing mutation in CFTR, $\triangle F 508$, is retained in the ER due to a folding defect, therapies that allow $\Delta F 508$ to escape the ER are being intensely investigated $(11,12,64)$. Importantly, multiple labs have demonstrated that rescued $\Delta F 508$ CFTR can reach the cell surface where it has chloride channel activity (4). However, $\triangle F 508$ CFTR is cleared from the cell surface and degraded much more rapidly than WT CFTR (65). Therefore, an understanding of the mechanisms that regulate the stability of mature CFTR will provide important insights into therapeutic strategies aimed at rescuing $\triangle F 508$ CFTR. It is clear from these previous results that the maintenance of the plasma membrane pool of CFTR involves a complex set of regulatory interactions that govern aspects of trafficking, anchoring, internalization, and endocytic recycling. Direct and indirect proteinprotein interactions with $\mathrm{PDZ}$ protein, clathrin subunits, small GTPases, myosins, and syntaxins have all been shown to regulate these aspects of CFTR biology (reviewed in ref. 66). Here we demonstrate that FLNs are a key element of this regulatory network by anchoring CFTR on the plasma membrane and stabilizing the mature protein. In future studies, it will be important to assess how these accessory proteins coordinately maintain the plasma membrane pool of CFTR.

\section{Methods}

Plasmids. CFTR mammalian expression constructs were prepared in pcDNA3.1(+). All CFTR point mutants used in this study were generated by site-directed mutatagenesis using the QuikChange XL Kit (Stratagene) and sequenced at the University of North Carolina DNA sequencing facil- ity. Exotope CFTR (referred to as HA-CFTR) was generated by introducing an HA tag into a modified second extracellular loop of CFTR as described by Gentzsch et al. (53).

Cell culture. HEK293, HeLa, and BHK cells were cultured in DMEM supplemented with 10\% FBS and penicillin/streptomycin. M2 and A7 cells were cultured as previously described (39). BHK cells stably expressing HA-CFTR were grown in selection media supplemented with $500 \mu \mathrm{g} / \mathrm{ml}$ mexthotraxate. Calu-3 cells were maintained as described previously (67). WD-HBE cells were isolated from freshly excised bronchial specimens from normal subjects and cultured as described previously (67). Isolation of WD-HBE cultures was approved by the University of North Carolina Institutional Review Board. Transient transfections were performed using Effectene (QIAGEN) or Lipofectamine 2000 (Invitrogen) according to the manufacturer's suggestions. Assays were carried out $24-48$ hours after transfection. HA-CFTR was expressed in M2 and A7 cells using adenovirus generated by the Cystic Fibrosis Center Molecular Biology Core Facility at an MOI of 20.

Antibodies and other reagents. CFTR monoclonal antibodies 293 and 570 (used for Western blots) and CFTR 570 cross-linked to Protein G Dynabeads (Invitrogen; used for immunoprecipitation) were provided by J. Riordan (University of North Carolina). Cell surface labeling of HA-CFTR was performed using HA monoclonal antibody HA.11 (Covance). Full-legnth FLN-A was analyzed using monoclonal antibodies 1678 and 1680 (Chemicon International).

Affinity purification of CFTR-interacting proteins. Peptides corresponding to residues 1-25 of CFTR were synthesized followed by a serine-glycine-serine-gylcine (SGSG) linker region and a C-terminal lysine residue coupled to biotin (Genemed Synthesis and Tufts University Peptide Core Facility). The $\mathrm{CFTR}_{1-25}$ or $\mathrm{CFTR}_{1-25 / \mathrm{S} 13 \mathrm{~F}}$ peptides were used to affinity purify CFTRbinding proteins as described by Thelin et al. (67), with the following exceptions. Bound proteins were eluted with $100 \mu \mathrm{l}$ of $1 \times$ Laemmli sample buffer and resolved by SDS-PAGE. Gels were stained with Coomassie blue per the University of North Carolina-Duke Michael Hooker Proteomics Center standard procedures (http://proteomics.unc.edu/protocol.shtml). Visible bands were excised and prepared for MS analysis by MALDI-MS/MS as described previously (68).

CFTR immunoprecipitation. CFTR immunoprecipitation experiments were performed as described previously (67). Briefly, CFTR was immunoprecipitated from cell membranes solubilized in binding buffer $(50 \mathrm{mM}$ Tris-Cl, pH 7.4, $150 \mathrm{mM} \mathrm{NaCl}, 0.1 \%$ Triton-X 100) prepared from Calu-3 or HEK293 cells using the CFTR 570 antibody covalently bound to Protein-G Dynabeads (Invitrogen). Bound proteins were washed 4 times for 5 minutes per wash in binding buffer. Bound proteins were eluted with $1 \times$ Laemmli buffer, resolved by SDS-PAGE, and analyzed by Western blot.

Immunohistochemistry. Conditions for immunohistochemical analyses in Calu-3 cells and WD-HBE cultures were as previously described $(69,70)$. All confocal microscopy was performed in the Michael Hooker Microscopy Facility.

Pulse-chase analysis. WT, S13F, S13A, and $\Delta$ F508 CFTRs were transiently expressed in HEK293 cells. Twenty-four hours after transfection, the cells were starved in cysteine/methionine-free DMEM for 30 minutes. We incubated $250 \mu \mathrm{Ci} / \mathrm{ml}^{35} \mathrm{~S}$-promix (GE HealthCare) with the cells for an additional 30 minutes. The labeling media was removed, and the cells were chased with cold DMEM supplemented with $10 \%$ FBS and incubated at $37^{\circ} \mathrm{C}$ for the chase periods indicated in Figure 2A and Figure 9B. Cells were recovered by incubating with $15 \mathrm{mM}$ sodium citrate and pelleted by centrifugation. CFTR was immunoprecipitated from whole cell lysates as described above. Immunoprecipitates were resolved by SDS-PAGE, and the gels were dried and visualized by phosphorimager analysis. For lysosomal inhibitor studies, $10 \mu \mathrm{g} / \mathrm{ml}$ leupeptin was included in the culture media. All data were quantitated using ImageQuant TL software (GE HealthCare). 
CFTR surface labeling and ELISAs. HA-CFTR proteins were transiently expressed in HeLa or BHK cells grown on glass coverslips (immunofluorescence) or 24-well plates (ELISAs). Cells were fixed with 4\% PFA, blocked for 2 hours in blocking buffer (PBS with $2 \mathrm{mg} / \mathrm{ml} \mathrm{BSA}, 4 \%$ nonfat milk, and $1 \%$ fish gelatin), labeled in anti-HA antibodies in blocking buffer for 2 hours, washed 3 times for 10 minutes per wash, and labeled with either Alexa Fluor 488 (Immunofluorescence; Invitrogen), HRP-conjugated (ELISAs; Jackson ImmunoResearch Laboratories Inc.), or IRDye-680 (ELISAs; LI-COR Biosciences) secondary antibodies. Surface CFTR was analyzed in the absence of detergents, while total CFTR was analyzed in cells permeabilized with $0.1 \%$ Triton-X 100. For surface ELISAs, labeled cells were incubated with the HRP substrate ABTS (Pierce Biotechnology) for 30 minutes at room temperature and analyzed by a microplate reader at $405 \mathrm{~nm}$ or analyzed using an Odyssey infrared imaging system (LI-COR Biosciences).

SPT assays. HA-CFTR was expressed in HeLa cells, and SPT experiments were performed as described previously (47). Briefly, cell surface CFTR was labeled using biotinylated HA antibodies followed by anti-biotin-conjugated gold (40 $\mathrm{nm}$ in diameter). Gold-labeled CFTR was analyzed by computer-enhanced video microscopy as described previously (47). CFTR proteins were imaged in brightfield mode for 1,800 frames/particle at 30 Hz. Movies were analyzed by ISEE software (version 3.1; Inovision), and all trajectories were visually inspected to ensure correct tracking.

CFTR internalization assays. HeLa and BHK cells expressing HA-CFTR proteins were chilled to $4{ }^{\circ} \mathrm{C}$ on ice and incubated with $\mathrm{HA}$ antibodies diluted in serum-free DMEM $(0.05 \mu \mathrm{g} / \mu \mathrm{l})$ for 10 minutes. Unbound antibodies were washed off, and the cells were returned to $37^{\circ} \mathrm{C}$ for various times to allow the CFTR/antibody complexes to internalize. For immunofluorescence assays, cells were fixed in $4 \%$ PFA, incubated in blocking solution with $0.1 \%$ Triton-X 100, and labeled with Alexa Fluor 488-conjugated anti-mouse antibodies. Lysosomes were labeled by incubating cells in $50 \mathrm{nM}$ lysotracker red dye (Invitrogen) for 30 minutes. For endocytosis experiments, unpermeabilized cells were fixed in 4\% PFA, incubated with IRDye-680 secondary antibodies, and analyzed using the Odyssey infrared imaging system.

Peptide transfection. To disrupt the CFTR-FLN interaction in situ, short competitive peptides were introduced into cells using a lipid delivery system. Specifically, nonbiotinylated $\mathrm{CFTR}_{1-25}$ or $\mathrm{CFTR}_{1-25 / \mathrm{S} 13 \mathrm{~F}}$ peptides were introduced into BHK cells stably expressing HA-CFTR with the Pro-Ject transfection system (Pierce Biotechnology). Complexes were formed by incubating $5 \mu \mathrm{g}$ of CFTR peptides with $5 \mu$ l of the Pro-Ject reagent in a total volume of $40 \mu \mathrm{l}$ HEPES-buffered saline (10 $\mathrm{mM}$ HEPES, $150 \mathrm{mM} \mathrm{NaCl}$, $\mathrm{pH}$ 7.0). Because the peptides cannot be visualized, Alexa Fluor 488-conjugated $F\left(a b^{\prime}\right)$ fragments were mixed with the peptides at a molar ratio of $4: 1$ [peptide/F( $\left(\mathrm{ab}^{\prime}\right)$ fragment]. Under these conditions, we achieved consistent delivery of the fluorescent probe to cells. However, the transfection efficiency was generally less than $10 \%$. In order to analyze the effects of the CFTR peptides, we labeled the cell surface pool of CFTR using Alexa Fluor 594 secondary antibodies. For each condition, the presence or absence of CFTR surface expression was assessed in transfected cells. For each experiment, 50 cells were counted in a double-blind fashion with $n=4$ for each condition.

Statistics. Quantitative data including densitometry, phosphorimager analysis, and ELISAs were analyzed by 1-way ANOVA using GraphPad Prism 4. Multiple comparisons were made using Tukey's post-hoc test. For all graphs, error bars represent \pm SEM. $P$ values less than 0.05 were considered significant.

\section{Acknowledgments}

We thank Michael Knowles (University of North Carolina), Jeffery Wine (Stanford University), Noreen Henig (Stanford University), and Gary Cutting (Johns Hopkins University) for helpful discussions regarding S13F patients; Gary Thomas (Oregon Health Sciences University) for providing M2 and A7 cells; John Riordan (University of North Carolina) for providing antibodies and plasmids; and Wendy Salmon (University of North Carolina) for help with microscopy. We thank the Milgram lab for technical assistance and Anthony Barnes and Caleb Hodson for helpful discussions. This work was supported by American Heart Association grant 0315363 U (to W.R. Thelin), the Cystic Fibrosis Foundation, and NIH grant R01HL080561 (to S.L. Milgram).

Received for publication September 18, 2006, and accepted in revised form November 21, 2006.

Address correspondence to: Sharon Milgram, University of North Carolina at Chapel Hill, 522 Taylor Hall, Chapel Hill, North Carolina 27599, USA. Phone: (919) 966-9792; Fax: (919) 966-1856; E-mail: milg@med.unc.edu.
1. Welsh, M.J., and Smith, A.E. 1993. Molecular mechanisms of CFTR chloride channel dysfunction in cystic fibrosis. Cell. 73:1251-1254.

2. Zielenski, J., and Tsui, L.C. 1995. Cystic fibrosis: genotypic and phenotypic variations. Annu. Rev. Genet. 29:777-807.

3. Haardt, M., Benharouga, M., Lechardeur, D., Kartner, N., and Lukacs, G.L. 1999. C-terminal truncations destabilize the cystic fibrosis transmembrane conductance regulator without impairing its biogenesis. A novel class of mutation. J. Biol. Chem. 274:21873-21877.

4. Denning, G.M., et al. 1992. Processing of mutant cystic fibrosis transmembrane conductance regulator is temperature-sensitive. Nature. 358:761-764.

5. Du, K., Sharma, M., and Lukacs, G.L. 2005. The DeltaF508 cystic fibrosis mutation impairs domaindomain interactions and arrests post-translational folding of CFTR. Nat. Struct. Mol. Biol. 12:17-25.

6. Anderson, M.P., and Welsh, M.J. 1992. Regulation by ATP and ADP of CFTR chloride channels that contain mutant nucleotide-binding domains. Science. 257:1701-1704.

7. Drumm, M.L., et al. 1991. Chloride conductance expressed by delta F508 and other mutant CFTRs in Xenopus oocytes. Science. 254:1797-1799.

8. Jurkuvenaite, A., et al. 2006. Mutations in the amino terminus of the cystic fibrosis transmem- brane conductance regulator enhance endocytosis. J. Biol. Chem. 281:3329-3334.

9. Silvis, M.R., et al. 2003. A mutation in the cystic fibrosis transmembrane conductance regulator generates a novel internalization sequence and enhances endocytic rates. J. Biol. Chem. 278:11554-11560.

10. Ward, C.L., Omura, S., and Kopito, R.R. 1995. Degradation of CFTR by the ubiquitin-proteasome pathway. Cell. 83:121-127.

11. Van Goor, F., et al. 2006. Rescue of DeltaF508CFTR trafficking and gating in human cystic fibrosis airway primary cultures by small molecules. Am. J. Physiol. Lung Cell. Mol. Physiol. 290:L1117-L1130.

12. Pedemonte, N., et al. 2005. Small-molecule correctors of defective $\Delta F 508$-CFTR cellular processing identified by high-throughput screening. J. Clin. Invest. 115:2564-2571. doi:10.1172/JCI24898.

13. Prince, L.S., et al. 1999. Efficient endocytosis of the cystic fibrosis transmembrane conductance regulator requires a tyrosine-based signal. J. Biol. Chem. 274:3602-3609.

14. Zhang, H., et al. 2002. Cysteine string protein interacts with and modulates the maturation of the cystic fibrosis transmembrane conductance regulator. J. Biol. Chem. 277:28948-28958.

15. Zhang, H., et al. 2006. Cysteine string protein monitors late steps in cystic fibrosis transmembrane conductance regulator biogenesis. J. Biol. Chem.
281:11312-11321.

16. Chang, X.B., et al. 1999. Removal of multiple arginine-framed trafficking signals overcomes misprocessing of delta F508 CFTR present in most patients with cystic fibrosis. Mol. Cell. 4:137-142.

17. Hegedus, T., et al. 2006. F508del CFTR with two altered RXR motifs escapes from ER quality control but its channel activity is thermally sensitive. Biochim. Biophys. Acta. 1758:565-572.

18. Cormet-Boyaka, E., et al. 2002. CFTR chloride channels are regulated by a SNAP-23/syntaxin 1A complex. Proc. Natl. Acad. Sci. U. S. A. 99:12477-12482.

19. Naren, A.P., et al. 1997. Regulation of CFTR chloride channels by syntaxin and Munc18 isoforms. Nature. 390:302-305.

20. Chang, S.Y., et al. 2002. Mechanisms of CFTR regulation by syntaxin $1 \mathrm{~A}$ and PKA. J. Cell Sci. 115:783-791.

21. Ganeshan, R., Di, A., Nelson, D.J., Quick, M.W., and Kirk, K.L. 2003. The interaction between syntaxin $1 \mathrm{~A}$ and cystic fibrosis transmembrane conductance regulator $\mathrm{Cl}^{-}$channels is mechanistically distinct from syntaxin 1A-SNARE interactions. J. Biol. Chem. 278:2876-2885.

22. Naren, A.P., et al. 2000. Syntaxin $1 \mathrm{~A}$ is expressed in airway epithelial cells, where it modulates CFTR $\mathrm{Cl}(-)$ currents. J. Clin. Invest. 105:377-386.

23. Naren, A.P., Quick, M.W., Collawn, J.F., Nelson, 
D.J., and Kirk, K.L. 1998. Syntaxin 1A inhibits CFTR chloride channels by means of domain-specific protein-protein interactions. Proc. Natl. Acad. Sci. U. S. A. 95:10972-10977.

24. Peters, K.W., Qi, J., Watkins, S.C., and Frizzell, R.A. 1999. Syntaxin 1A inhibits regulated CFTR trafficking in xenopus oocytes. Am. J. Physiol. 277:C174-C180

25. Fu, J., Ji, H.L., Naren, A.P., and Kirk, K.L. 2001. A cluster of negative charges at the amino terminal tail of CFTR regulates ATP-dependent channel gating. J. Physiol. 536:459-470.

26. Naren, A.P., et al. 1999. CFTR chloride channel regulation by an interdomain interaction. Science. 286:544-548.

27. Cormet-Boyaka, E., et al. 2004. Rescuing cystic fibrosis transmembrane conductance regulator (CFTR)-processing mutants by transcomplementation. Proc. Natl. Acad. Sci. U. S. A. 101:8221-8226.

28. Stossel, T.P., et al. 2001. Filamins as integrators of cell mechanics and signalling. Nat. Rev. Mol. Cell Biol. 2:138-145.

29. Wang, K., and Singer, S.J. 1977. Interaction of filamin with f-actin in solution. Proc. Natl. Acad. Sci. U. S. A. 74:2021-2025

30. Feng, Y., and Walsh, C.A. 2004. The many faces of filamin: a versatile molecular scaffold for cell motility and signalling. Nat. Cell Biol. 6:1034-1038.

31. Leoni, G.B., Pitzalis, S., Tonelli, R., and Cao, A. 1998. Identification of a novel mutation (S13F) in the CFTR gene in a CF patient of Sardinian origin. Hum. Mutat. 11:337.

32. Wine, J.J., Kuo, E., Hurlock, G., and Moss, R.B. 2001. Comprehensive mutation screening in a cystic fibrosis center. Pediatrics. 107:280-286.

33. Cheng, S.H., et al. 1990. Defective intracellular transport and processing of CFTR is the molecular basis of most cystic fibrosis. Cell. 63:827-834.

34. Seibert, F.S., et al. 1997. Disease-associated mutations in cytoplasmic loops 1 and 2 of cystic fibrosis transmembrane conductance regulator impede processing or opening of the channel. Biochemistry. 36:11966-11974

35. Seibert, F.S., et al. 1996. Disease-associated mutations in the fourth cytoplasmic loop of cystic fibrosis transmembrane conductance regulator compromise biosynthetic processing and chloride channel activity. J. Biol. Chem. 271:15139-15145.

36. Anilkumar, G., et al. 2003. Prostate-specific membrane antigen association with filamin A modulates its internalization and NAALADase activity. Cancer Res. 63:2645-2648.

37. Feng, S., Resendiz, J.C., Lu, X., and Kroll, M.H. 2003. Filamin A binding to the cytoplasmic tail of glycoprotein Ibalpha regulates von Willebrand factorinduced platelet activation. Blood. 102:2122-2129.

38. Lin, R., Karpa, K., Kabbani, N., Goldman-Rakic, P., and Levenson, R. 2001. Dopamine D2 and D3 receptors are linked to the actin cytoskeleton via interaction with filamin A. Proc. Natl. Acad. Sci. U. S. A. 98:5258-5263.

39. Liu, G., et al. 1997. Cytoskeletal protein ABP-280 directs the intracellular trafficking of furin and modulates proprotein processing in the endocytic pathway. J. Cell Biol. 139:1719-1733.

40. Sampson, L.J., Leyland, M.L., and Dart, C. 2003. Direct interaction between the actin-binding protein filamin-A and the inwardly rectifying potassium channel, Kir2.1. J. Biol. Chem. 278:41988-41997.

41. Seck, T., Baron, R., and Horne, W.C. 2003. Binding of filamin to the $\mathrm{C}$-terminal tail of the calcitonin receptor controls recycling. J. Biol. Chem. 278:10408-10416.

42. Zhang, M., and Breitwieser, G.E. 2005. High affinity interaction with filamin A protects against calcium-sensing receptor degradation. J. Biol. Chem. 280:11140-11146.

43. Lambert, M., Choquet, D., and Mege, R.M. 2002. Dynamics of ligand-induced, Rac1-dependent anchoring of cadherins to the actin cytoskeleton. J. Cell Biol. 157:469-479.

44. Sheets, E.D., Lee, G.M., Simson, R., and Jacobson, K. 1997. Transient confinement of a glycosylphosphatidylinositol-anchored protein in the plasma membrane. Biochemistry. 36:12449-12458.

45. Sako, Y., Nagafuchi, A., Tsukita, S., Takeichi, M., and Kusumi, A. 1998. Cytoplasmic regulation of the movement of E-cadherin on the free cell surface as studied by optical tweezers and single particle tracking: corralling and tethering by the membrane skeleton. J. Cell Biol. 140:1227-1240.

46. Kusumi, A., and Sako, Y. 1996. Cell surface organization by the membrane skeleton. Curr. Opin. Cell Biol. 8:566-574.

47. Chen, Y., Thelin, W.R., Yang, B., Milgram, S.L., and Jacobson, K. 2006. Transient anchorage of crosslinked glycosyl-phosphatidylinositol-anchored proteins depends on cholesterol, Src family kinases, caveolin, and phosphoinositides. J. Cell Biol. 175:169-178

48. Prince, L.S., Workman, R.B., Jr., and Marchase, R.B. 1994. Rapid endocytosis of the cystic fibrosis transmembrane conductance regulator chloride channel. Proc. Natl. Acad. Sci. U. S. A. 91:5192-5196.

49. Peter, K., et al. 2002. Ablation of internalization signals in the carboxyl-terminal tail of the cystic fibrosis transmembrane conductance regulator enhances cell surface expression. J. Biol. Chem. 277:49952-49957.

50. Bradbury, N.A., et al. 1999. Characterization of the internalization pathways for the cystic fibrosis transmembrane conductance regulator. Am. J. Physiol. 276:L659-L668.

51. Bradbury, N.A., Cohn, J.A., Venglarik, C.J., and Bridges, R.J. 1994. Biochemical and biophysical identification of cystic fibrosis transmembrane conductance regulator chloride channels as components of endocytic clathrin-coated vesicles. J. Biol. Chem. 269:8296-8302.

52. Lukacs, G.L., Segal, G., Kartner, N., Grinstein, S., and Zhang, F. 1997. Constitutive internalization of cystic fibrosis transmembrane conductance regulator occurs via clathrin-dependent endocytosis and is regulated by protein phosphorylation. Biochem. J. 328:353-361.

53. Gentzsch, M., et al. 2004. Endocytic trafficking routes of wild type and DeltaF508 cystic fibrosis transmembrane conductance regulator. Mol. Biol.
Cell. 15:2684-2696

54. Gravante, B., Barbuti, A., Milanesi, R., Zappi, I., Viscomi, C., and DiFrancesco, D. 2004. Interaction of the pacemaker channel HCN1 with filamin A. J. Biol. Chem. 279:43847-43853.

55. Bates, I.R., et al. 2006. Membrane lateral diffusion and capture of CFTR within transient confinement zones. Biophys. J. 91:1046-1058.

56. Chen, Y., Yang, B., and Jacobson, K. 2004. Transient confinement zones: a type of lipid raft? Lipids. 39:1115-1119.

57. Short, D.B., et al. 1998. An apical PDZ protein anchors the cystic fibrosis transmembrane conductance regulator to the cytoskeleton. J. Biol. Chem. 273:19797-19801.

58. Sun, F., et al. 2000. E3KARP mediates the association of ezrin and protein kinase A with the cystic fibrosis transmembrane conductance regulator in airway cells. J. Biol. Chem. 275:29539-29546.

59. Feng, S., Lu, X., and Kroll, M.H. 2005. Filamin A binding stabilizes nascent glycoprotein Ibalpha trafficking and thereby enhances its surface expression. J. Biol. Chem. 280:6709-6715.

60. Swiatecka-Urban, A., et al. 2002. PDZ domain interaction controls the endocytic recycling of the cystic fibrosis transmembrane conductance regulator. J. Biol. Chem. 277:40099-40105.

61. Sharma, M., et al. 2004. Misfolding diverts CFTR from recycling to degradation: quality control at early endosomes. J. Cell Biol. 164:923-933.

62. Head, B.P., et al. 2006. Microtubules and actin microfilaments regulate lipid raft/caveolae localization of adenylyl cyclase signaling components. J. Biol. Chem. 281:26391-26399.

63. Prat, A.G., et al. 1999. Actin filament organization is required for proper cAMP-dependent activation of CFTR. Am. J. Physiol. 277:C1160-C1169.

64. Yang, H., et al. 2003. Nanomolar affinity small molecule correctors of defective Delta F508-CFTR chloride channel gating. J. Biol. Chem. 278:35079-35085.

65. Swiatecka-Urban, A., et al. 2005. The short apical membrane half-life of rescued DeltaF508-cystic fibrosis transmembrane conductance regulator (CFTR) results from accelerated endocytosis of DeltaF508-CFTR in polarized human airway epithelial cells. J. Biol. Chem. 280:36762-36772.

66. Guggono, W.B., and Stanton, B.A. 2006. New insights into cystic fibrosis: molecular switches that regulate CFTR. Nat. Rev. Mol. Cell. Biol. 7:426-436.

67. Thelin, W.R., et al. 2005. The cystic fibrosis transmembrane conductance regulator is regulated by a direct interaction with the protein phosphatase 2A. J. Biol. Chem. 280:41512-41520.

68. Loiselle, D.R., et al. 2005. Improved protein identification through the use of unstained gels. J. Proteome Res. 4:992-997.

69. Kreda, S.M., et al. 2005. Characterization of wildtype and deltaF508 cystic fibrosis transmembrane regulator in human respiratory epithelia. Mol. Biol. Cell. 16:2154-2167.

70. Mohler, P.J., et al. 1999. Yes-associated protein 65 localizes p62(c-Yes) to the apical compartment of airway epithelia by association with EBP50. J. Cell Biol. 147:879-890. 